\title{
Micro- and Nano-Carrier Mediated Intra-Articular Drug Delivery Systems for the Treatment of Osteoarthritis
}

\author{
Zhiyue Zhang and Guihua Huang \\ School of Pharmaceutical Sciences, Shandong University, 44 West Wenhua Road, Jinan, Shandong 250012, China \\ Correspondence should be addressed to Guihua Huang, hgh2003@gmail.com
}

Received 11 March 2011; Accepted 24 April 2011

Academic Editor: Lifeng Dong

Copyright $\odot 2012$ Z. Zhang and G. Huang. This is an open access article distributed under the Creative Commons Attribution License, which permits unrestricted use, distribution, and reproduction in any medium, provided the original work is properly cited.

\begin{abstract}
The objective of this paper is to provide readers with current developments of intra-articular drug delivery systems. In recent years, although the search for a clinically successful ideal carrier is ongoing, sustained-release systems, such as polymeric micro- and nanoparticles, liposomes, and hydrogels, are being extensively studied for intra-articular drug delivery purposes. The advantages associated with long-acting preparations include a longer effect of the drug in the action site and a reduced risk of infection due to numerous injections consequently. This paper discusses the recent developments in the field of intra-articular sustained-release delivery systems for the treatment of osteoarthritis.
\end{abstract}

\section{Introduction}

Arthritis is a degenerative joint disease common among elderly people that causes progressive joint degeneration leading to chronic pain and reduced quality of life [1-4]. Osteoarthritis (OA) and rheumatoid arthritis (RA), two main kinds of arthritis, are among the most important inflammatory diseases, afflicting 40 million people (15\%) in the US alone in 1995 and expected to afflict 59.4 million (18.2\%) by the year 2020 [5]. And osteoarthritis $(\mathrm{OA})$ is the most prevalent musculoskeletal condition that causes joint pain. The incidence of $\mathrm{OA}$ increases with age, and approximately $100 \%$ of men and women at age $75-$ 79 years shows some signs of OA [6]. Current available treatment is of symptoms, directed to relieve the pain and regain function [7], but outcomes are limited. For example, oral administration of NSAID tablets relieves inflammation and pain but causes gastrointestinal adverse effects; other effective treatments are surgeries, but they will cause the second damage to the joint as well as high cost.

Local drug delivery strategies may provide for the development of more successful OA treatment outcomes that have potential to reduce local joint inflammation and joint destruction, offer pain relief, and restore patient activity levels and joint function [8]. Intra-articular drug delivery is very useful for treating local disease flareups, synovitis, and pain in joints. The Food and Drug Administration (FDA) has approved intra-articular hyaluronic acid formulations for osteoarthritis of the knee [9]. The intra-articular (IA) route of administration has potential for targeting drug delivery to affected tissues in treating arthritis, thereby minimizing the attendant side effects of systemically administered drugs [10].

However, current preparations of intra-articular drug delivery often require frequent injections that have a high financial burden, impaction to patient's quality of life, rapid degradation and clearance of injected pharmacologic agents, and also increase the risk of complications [11]. Micro- and nanocarrier-mediated drug delivery systems, including polymeric particles, liposome, and hydrogel, are well-established as methods for sustained release in intraarticular applications. These systems could prolong drug retention time, reduce the clearance of drug into joint cavity, and increase patient compliance as well as therapeutic effect of pharmaceutical agents. This process guaranteed a longer effect of the drug in the action site and consequently, a reduced risk of infection due to numerous injections [12]. Sustained therapeutic drug concentrations can also be achieved with intra-articular slow-release drug delivery 

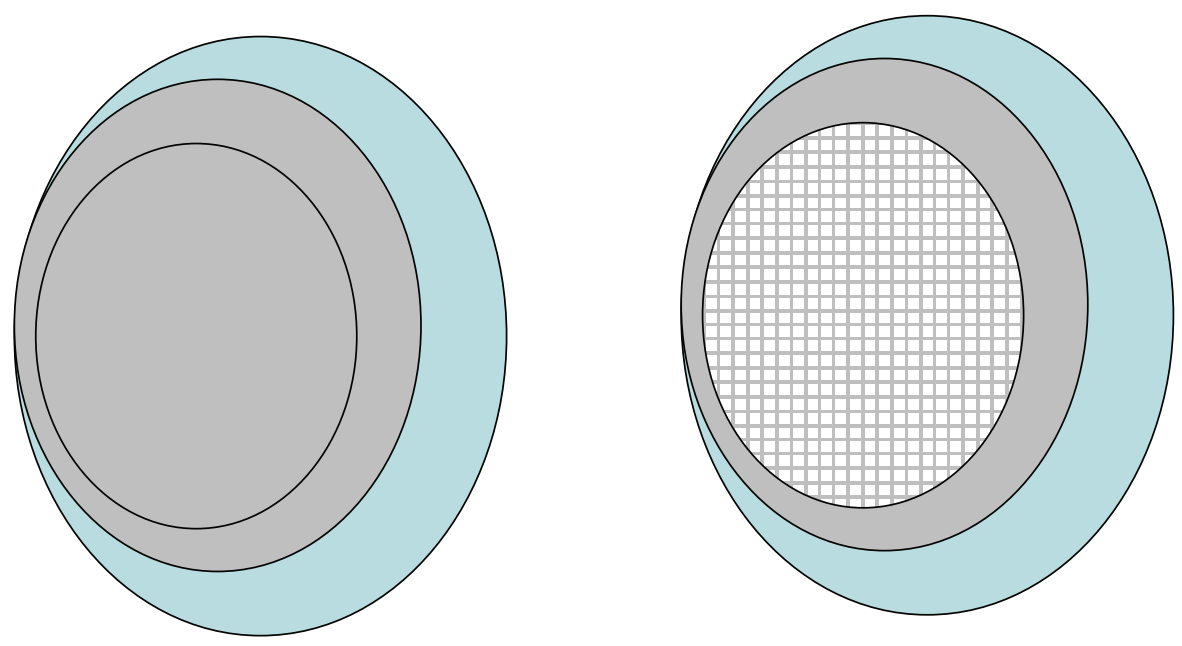

FIGURE 1: Schematic representation of a microsphere and a microcapsule. In microspheres, the whole particle consists of a continuous polymer network. Microcapsules present a core-shell structure with a liquid core surrounded by a polymer shell.

device, rather than repeated injections. It has been shown that sustained intra-articular drug concentration can be realized through coupling the desired drug to liposomes, microparticles, or hydrogels. The purpose of this paper is to summarize the current state of intra-articular longacting injection preparations applied to the treatment of osteoarthritis. The main goal in the future is to increase the residence time of the drug in the joint as well as improve its diffusion within the target tissue [13].

\section{Micro- and Nano-Carrier Mediated Drug Delivery Systems for IA Formulations}

2.1. Polymeric Particles. Biodegradable particles have proven to be very useful drug delivery systems and provide prolonged drug release and lasting delivery because of sustained release of the microencapsulated material. Due to their advantage of being biodegradable and biocompatible, polymeric micro- and nanocarriers in formulations of therapeutic drug delivery systems have gained widespread application. The drug is dispersed or encapsulated in a microparticle matrix, and, depending on the preparation method, microspheres or microcapsules can be obtained (Figure 1). Among the microparticulate systems, microspheres have a special importance since it is possible to target drugs and provide controlled release [14]. Meanwhile, micro- and nanoparticulate drug delivery systems have been studied extensively using various kinds of biodegradable polymers during the past two decades [15].

Commonly used materials and their derivatives for preparing micro- and nanoparticles bifurcate natural materials including chitosan, gelatin, alginate, and other materials and synthetic materials, such as PLA(polylactide), PLGA(poly(lactic-co-glycolic acid)), PCL(polycaprolactone), and other synthetic ones [16]. Prolonged time of drug release and decreased toxicity and stimulation can be achieved via intra-articular controlled release system of polymeric particles [17].

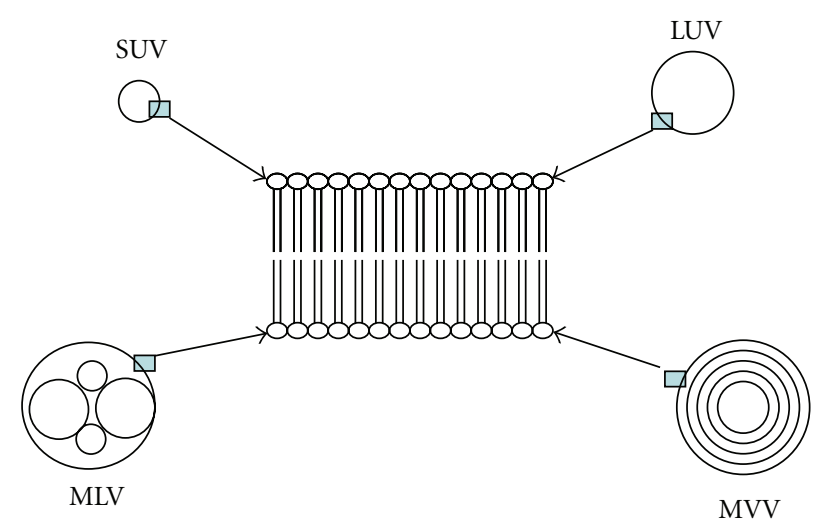

FIgure 2: The classification of liposomes. SUV: small unilamellar vesicles; LUV: large unilamellar vesicles; MLV: large multilamellar vesicles; MVV: multivesicular vesicles.

The most commonly used methods of microencapsulation encompass solvent extraction or evaporation, coacervation, and spray drying [18]. The composition and preparation methods of microcapsules were found to be closely related to drug release and microcapsule degradation. Meanwhile, the release profile was monophasic or biphasic depending on the formulation [19].

2.2. Liposomes. Liposomes are spherical vesicles or cavities made up of phospholipids that usually, but not by definition, contain a core of aqueous solution and have been proposed as efficient carriers for controlled drug delivery (Figure 2). They are able to entrap hydrophilic drugs in the large aqueous interior and lipophilic drugs inserted in the lipid bilayer [20]. Moreover, hydrophobic drugs such as dexamethasone are incorporated in the bilayer structure of liposomes, whereas hydrophilic drugs such as diclofenac are encapsulated in the internal aqueous chamber [21]. Derived from naturally occurring, biodegradable and nontoxic lipids, liposomes are 
good candidates for local targeting of therapeutic agents to the site of interest, while reducing systemic toxicity. And the residence of encapsulated drugs within the knee joint was greatly prolonged. For example, liposomal iohexol declined biexponentially with a terminal elimination half-life of 134 hours compared with free iohexol which was undetectable 3 hours after injection [20,22]. Liposomes are good candidates although a liposomal corticosteroid formulation containing dexamethasone-21-palmitate (Lipotalon) available in Germany is the only intra-articular liposomal product used in human patients [20].

Conventional methods of liposome manufacture can be said to involve four basic stages: drying down of lipids from organic solvents, dispersion of the lipids in aqueous media, purification of the resultant liposomes, and analysis of the final product [23] (Figure 3). In order to optimize the liposomal formulation for local use, liposomes with appropriate size, surface properties, and composition should be selected with respect to the site of administration, the disease, and the drug used. In a word, a balance is of importance between the stability of the liposomes and their ability to deliver drugs [24].

2.3. Hydrogels. Since the pioneering work of Wichterle and Lim in 1960 on crosslinked HEMA hydrogels, hydrogels have been of great interest to biomaterial scientists for many years because of their hydrophilic character and potential to be biocompatible [25]. Hydrogels are hydrophilic polymer networks which may absorb from 10-20\% (an arbitrary lower limit) up to thousands of times their dry weight in water and may be chemically stable or degrade and eventually disintegrate and dissolve. They are called "reversible" or "physical" gels when the networks are held together by molecular entanglements and/or secondary forces including ionic, H-bonding, or hydrophobic forces [26]. Both natural and synthetic materials have been used to form scaffolds. Naturally derived materials often have desirable biological properties and also possess limited mechanical strength or fast degradation profiles that may not be suitable for clinical applications. Synthetic polymers, although not as bioactive as natural scaffolds, can provide the necessary properties to produce scaffolds with desired controllable physical and chemical characteristics. Many kinds of synthetic hydrogel polymers, such as methyl methacrylate, poly(2-hydroxyethyl)methacrylate (polyHEMA) hydrogel, poly(vinyl alcohol) (PVA) hydrogel, and are developed to repair or replace the damaged articular cartilage [27]. Hydrogels are required to have acceptable biodegradability and biomedical purposes. Figure 4 revealed a great variety of crossing approaches developed to prepare desired hydrogels.

Not only does sustained drug release reduce administration times and undesired side effects, but also it improves the patients' compliance and comfort significantly. When applied in a drug delivery system, the injectable drug/polymer formulation can be free of any organic solvent in the drug-loading process (an organic solvent might denature labile therapeutic agents like proteins), and the rate of drug release is easily adjusted via altering the material

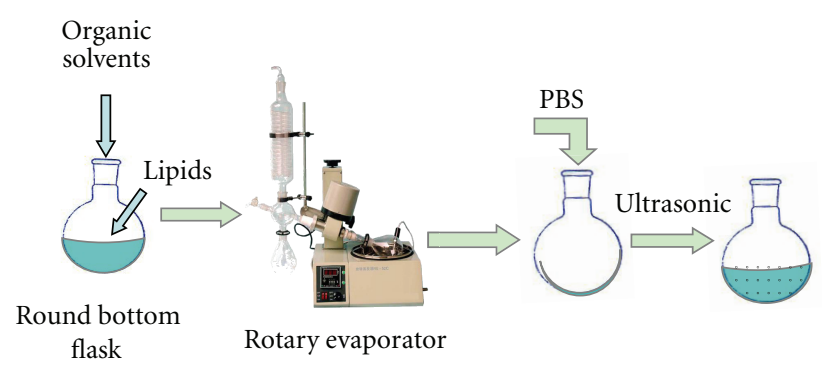

FIGURE 3: Conventional liposome preparation method: film dispersion method.

properties. These hydrogel formulations are useful for parenteral and topical injection for a site-specific action [28]. Their affinity to absorb water is attributed to the presence of hydrophilic groups such as $-\mathrm{OH},-\mathrm{CONH}-,-\mathrm{CONH}_{2}-$, and $-\mathrm{SO}_{3} \mathrm{H}$ in polymers forming hydrogel structures [29]. In contrast, the polymeric networks of hydrophobic characteristics (e.g., poly(lactic acid) (PLA) or poly(lactide-coglycolide) (PLGA)) are limiting water-absorbing capacities $(<5-10 \%)$, and the polymer is thus hydrated to different degrees (sometimes more than $90 \% \mathrm{wt}$.) depending on the nature of the aqueous environment and polymer composition due to the contribution of these groups and domains in the network [30].

\section{NSAIDs Delivery Systems}

Nonsteroidal anti-inflammatory drugs (NSAIDs) are a series of drugs having anti-inflammatory, analgesic, and antipyretic effects which alleviate pain by counteracting the cyclooxygenase $(\mathrm{COX})$ enzyme which synthesizes prostaglandins, creating inflammation [31]. In whole, the NSAIDs prevent the prostaglandins from ever being synthesized, reducing or eliminating the pain [32].

NSAIDs can reduce short-term pain in osteoarthritis of the knee slightly better than placebo, but the current analysis does not support long-term use of NSAIDs for this condition. As serious adverse effects are associated with oral NSAIDs, only limited use can be recommended [33]. Cyclo-oxygenase-2 (COX 2) inhibitors are selective types of nonsteroidal anti-inflammatory drugs (NSAIDs) developed for the treatment of acute inflammation in joints. Although evidence shows that COX 2 inhibitors are as effective as traditional NSAIDs in relieving pain [34], they decrease arthritis without the gastrointestinal side effects associated with traditional NSAIDs.

Intra-articular administration of NSAIDs could be an alternative to delivery administration which avoids their devastating effects. However, the short synovial half-life of NSAIDs would require frequent injections to maintain therapeutic intra-articular levels. For this reason, sustained drug delivery devices are needed to offer an excellent alternative to multiple intra-articular injections [35]. The working hypothesis was that increased NSAIDs efficacy and alleviation of adverse effects can be achieved by local administration of a new slow-release NSAID-carrier formulation. 


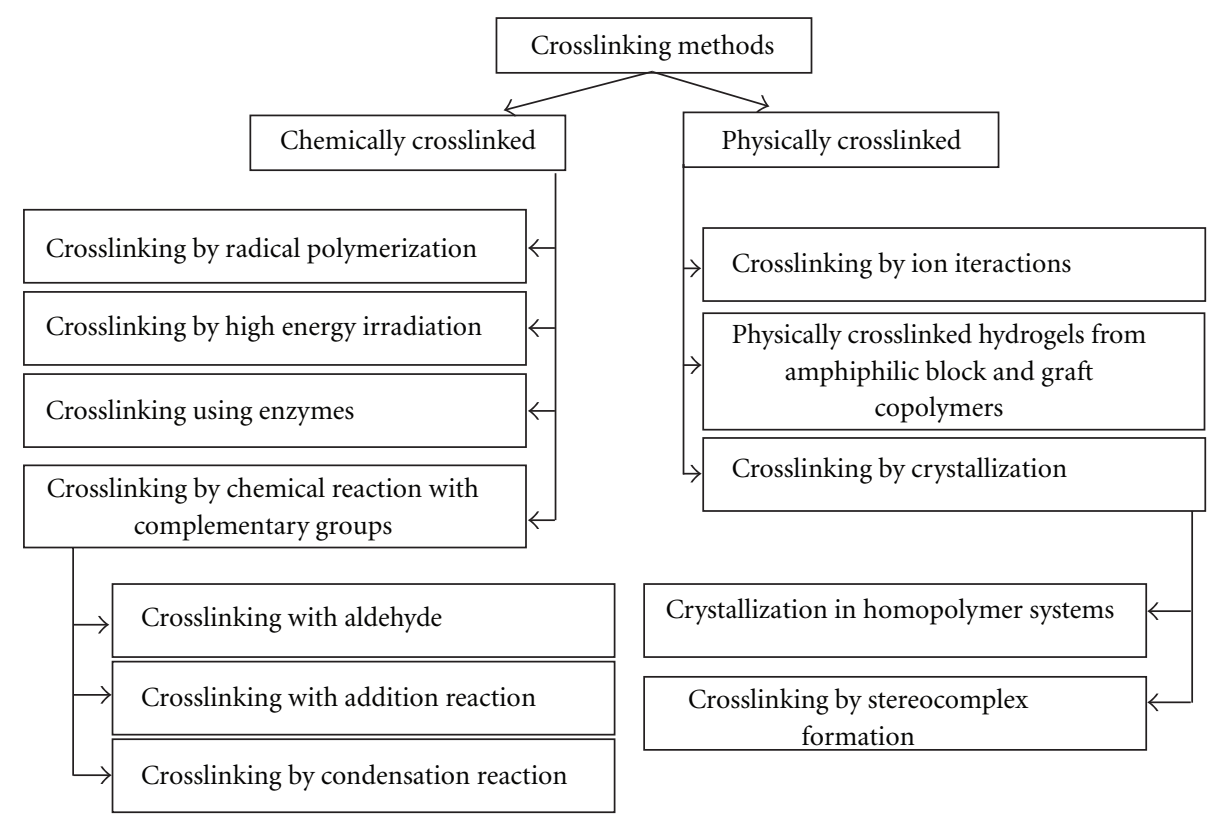

Figure 4: Novel crosslinking methods used in hydrogels [30].

3.1. Polymer Particles. Polymer particles loaded with an NSAID (such as diclofenac), destined for intra-articular administration, have been developed using different polymers such as chitosan, PLA, PLGA, and Poli $\delta$-valerolactona (PV) [36]. In the present study, controlled release parenteral formulations of diclofenac sodium were prepared for intraarticular administration [15].

Diclofenac is a nonsteroidal anti-inflammatory drug (NSAID) advocated for use in painful and inflammatory rheumatic and certain nonrheumatic conditions. It is available in a number of administration forms which can be given orally, rectally, or intramuscularly. Conveniently, dosage adjustments are not required in the elderly or in those patients with renal or hepatic impairment. The drug has a relatively short elimination half-life, which limits the potential for drug accumulation. The efficacy of diclofenac in numerous clinical trials is equivalent to that of the many newer and established NSAIDs with which it has been compared [37]. Available data suggest that, in patients with osteoarthritis, diclofenac sodium is comparable in efficacy and tolerability with naproxen, ibuprofen, sulindac, and diflunisal. As oral diclofenac is generally given in 3 divided daily doses, it may be at a disadvantage relative to less frequent administration with naproxen, diflunisal, and sulindac in osteoarthritis, although there is some evidence of diclofenac's efficacy when administered twice daily or once daily as a slow-release tablet [38].

Saravanan et al. [39] have previously reported on the targeting of diclofenac sodium in joint inflammation using gelatin magnetic microspheres which focuses on the formulation of gelatin microspheres for intra-articular administration to overcome complications in the administration of magnetic microspheres and achieve higher targeting efficiency. Drug-loaded microspheres were prepared by the emulsification/crosslinking method, characterized by size distribution, drug loading, scanning electron microscopy (SEM), Fourier transform infrared (FT-IR) spectroscopy, differential scanning calorimetry (DSC), X-ray diffraction (XRD), gas chromatography, and in vitro release studies along with targeting efficiency of microspheres that was studied in vivo in rabbits. The microspheres showed drug loading of $9.8,18.3$, and $26.7 \% \mathrm{w} / \mathrm{w}$ with an average size range of 37-46 $\mu \mathrm{m}$ depending upon the drug-polymer ratio. They were spherical in nature and free from surface drug as evidenced by the SEM photographs and the absence of drug-polymer interaction, and the amorphous nature of entrapped drug was revealed by FT-IR, DSC, and XRD. Gas chromatography confirms the absence of residual glutaraldehyde. The formulated microspheres could prolong the drug release up to 30 days in vitro. About 81.2 and $43.7 \%$ of administered drug in the microspheres were recovered from the target joint after 1 and 7 days of post intra-articular injection, respectively, revealing good targeting efficiency. The microspheres prepared were able to prolong the drug release over 24-30 days, and the application of sonication during in vitro release study has slightly increased the release rate. After intra-articular administration of microspheres, $77.7 \%$ of injected dose was recovered at the target site which revealed good targeting efficiency [40].

H. P. Thakkar and R. R. Murthy have compared the characteristics of the microspheres of chitosan prepared using two different crosslinking agents: formaldehyde and glutaraldehyde crosslinking and simple heat treatment. Chitosan microspheres were prepared by emulsification crosslinking method and characterized for entrapment efficiency, particle size, in vitro drug release, and surface morphology studied by scanning electron microscopy (SEM). The entrapment efficiency of the glutaraldehyde 
and formaldehyde crosslinked microspheres was significantly higher $(P<.05)$ than the heat-crosslinked ones which showed the fastest release in vitro drug release [41]. Meanwhile, in vitro drug release studies indicated that the microspheres crosslinked using glutaraldehyde showed slower release rate than those crosslinked with formaldehyde.

3.2. Liposomes. In the case of arthritic diseases, it has been reported that the intra-articular administration of antiinflammatory drugs encapsulated in liposomes shows prolonged residence in the joint and reduction of inflammation $[42,43]$. For example, liposome-entrapped methotrexate injected intra-articularly was 10 -fold more potent than the free drug in suppressing the development of arthritis. Liposomes are used to encapsulate both hydrophilic and hydrophobic small molecules as well as macromolecules such as proteins and genes.

Free lactoferrin disappeared rapidly from the injected joint, with two-thirds lost by $2 \mathrm{~h}$ and only $2 \%$ remaining at $24 \mathrm{~h}$. However, entrapment in positively charged liposomes markedly enhanced retention time, with close to $50 \%$ still present after $6 \mathrm{~h}$ and $15 \%$ at $24 \mathrm{~h}$. Surprisingly, lactoferrin entrapped in $\mathrm{pH}$-sensitive negatively charged liposomes was lost from the joint even more rapidly than the free protein, being virtually undetectable by $24 \mathrm{~h}$ [44].

To increase NSAID efficacy and alleviate the adverse effects, Inbar Elron-Gross prepared a new slow-release NSAID-carrier formulation by local administration. Diclofenac was the test NSAID, and collagomers-novel vesicularshaped microparticles based on collagen-lipid conjugateswere the carriers. Collagomers were stable in simulated synovial fluid and showed high-efficiency drug encapsulation $(85 \%)$ and slow drug release $(\tau 1 / 2=11$ days $)$ as well as high affinity to target cells ( $K d=2.6 \mathrm{nM}$ collagen) [45].

\section{Glucocorticoids Delivery Systems}

Glucocorticoids are potent drugs that have a multitude of pharmacological actions both at genomic and nongenomic levels. It was suggested that intra-articular steroids often ameliorate acute exacerbations of knee osteoarthritis associated with significant effusions, symptomatic involvement of inter joint and other nonweight-bearing articulations, synovial cysts, and lumbar facet arthropathy. Judicious use of intra-articular injections seldom produces significant adverse effects [46].

Many of the diseases in which glucocorticoids are routinely administered are featured by angiogenesis and enhanced capillary permeability, permitting targeted delivery using long-circulating drug delivery systems. By encapsulation of glucocorticoids in long-circulating liposomes, drug levels at the site of the pathology are markedly higher, increasing and prolonging therapeutic efficacy in models of osteoarthritis, multiple sclerosis, and cancer [47].

Glucocorticoids (GCs) provide one of the most effective treatments for osteoarthritis; however, their long-term use is marred by undesired side effects. Increased understanding of the mechanisms of glucocorticoid action enables the development of novel drugs, such as SEGRAs or liposomal glucocorticoids, to improve their benefit/risk ratio [48].

A variety of methods are currently used for intraarticular glucocorticoids (GCs) injection to the osteoarthritis, each with the goal of minimizing the potential for tissue damage. However, clinical tests have shown that intraarticular glucocorticoids are not devoid of side effects despite their net efficacy. For example, because of their crystalline structure, a condition referred to as crystal-induced arthritis was described in $10 \%$ of the patients receiving an intraarticular injection. Moreover, due to lymph drainage or macrophage uptake, the residence time of corticosteroid molecules in the joint is very short and frequent intraarticular injections are required in order to obtain a sustained clinical result. The complications of such frequent injections in terms of infections or joint instability remain severe although rare. All these aspects emphasize the need to develop drug delivery systems allowing the sustained release of the active substance in order to reduce the frequency of the intra-articular injections together with the related potential deleterious effects [11].

4.1. Polymer Particles. Glucocorticoids are widely used for the treatment of a number of diseases of nonendocrine origin. Owing to their strong anti-inflammatory actions, they have been used for rheumatism, asthma, inflammatory bowel diseases, as well as for the treatment of dermatitis and allergies [49]. The prime obstacle for conventional administration is the dose-dependent systemic side effects. Selective delivery of drugs to specific target sites would reduce the necessary dose of a given drug while still achieving an effective local concentration. As a result, the therapeutic efficacy would be increased. The use of particulate drug carriers is a possible strategy to achieve site-specific drug delivery and to decrease undesirable interactions at other body sites. In recent years, polymer particles have emerged as one of the most promising controlled release dosage forms, and several micro- and nanoparticle-based products for parenteral use are commercially available (e.g., Enantone, Decapeptyl Depot, and Pravidel) [50].

Horisawa et al. developed DL-lactide/glycolide copolymer (PLGA) nanospheres incorporating a water-soluble corticosteroid (betamethasone sodium phosphate (BSP)) with prolonged anti-inflammatory action. BSP-loaded nanospheres were prepared by an emulsion solvent diffusion method in oil (caprylate and caprate triglyceride). The drug release rate from the nanospheres in PBS was controlled by the molecular weight and the lactic/glycolic acid (LA/GA) ratio of the polymers. Sustained drug release occurred for over three-week in vitro release study demonstrated, and the joint swelling decreased significantly by administering BSP-loaded nanospheres during a 21-day period after intraarticular challenge in the antigen-induced arthritic rabbit. The histologic safety of nanospheres administration to inflamed synovial tissue was confirmed. Meanwhile, prolonged local anti-inflammatory action in joint diseases without biologic damage can be provided by the PLGA particulate system [51]. 
In Butoescu's study, for the aim of locally treating inflammatory conditions such as arthritis, SPIONs and the corticosteroid dexamethasone acetate (DXM) are coencapsulated into PLGA microparticles. The microparticles in the joint with an external magnet can be maintained due to the magnetic properties conferred by the SPIONs. As superparamagnetic microparticles present the double advantage of internalization by the synoviocytes and a prolonged drug action due to magnetically increased microparticle residence time in the joint, DXM-containing ones seem to be promising drug delivery systems for the local treatment of arthropathies. The encapsulation process did not affect the magnetic properties of SPIONs or their oxidation state. Moreover, DXM and SPIONs were completely embedded into the microparticles, as demonstrated by the zeta potential $[52,53]$.

4.2. Liposomes. Dexamethasone is a potent anti-inflammatory and immunosuppressive glucocorticoid used for the treatment of inflammatory and autoimmune conditions such as osteoarthritis, edema, and multiple myeloma, as an adjunct with chemotherapy, and for nasal and eye allergies. The encapsulation of dexamethasone in carrier systems such as liposomes and microspheres can diminish the adverse effects and reduce the total amount of drug required. Liposomal and microsphere dexamethasone formulations have been investigated for hypersensitivity pneumonitis [21].

There are a number of literature reports concerning liposome formulations of dexamethasone and other glucocorticoids [54]. However, the physicochemical properties of these liposomes vary considerably. U. Bhardwaj and D. J. Burgess investigated the effects of process including sonication and extrusion, and formulation parameters such as lipid type, incorporation of cholesterol and dexamethasone on the physicochemical properties and thermotropic behavior of dexamethasone-loaded liposomes. The similar mean diameter of all the nonextruded liposomes indicated that particle size appeared to be dependent on the initial film thickness, since the concentration and volume of the lipid solutions were kept constant for all liposome preparations. Sonication- and extrusion-induced interdigitation leads to a decrease in space between the acyl chains and the higher loss of dexamethasone from the extruded DPPC liposomes. Cholesterol incorporation has been reported to increase membrane stability, decrease permeability, and increase encapsulation of hydrophilic drugs. However, both cholesterol and dexamethasone have a similar hydrophobic steroidal molecular structure and compete for the same sites in the liposome membrane. Cholesterol is more lipophilic $(\log p \sim 7.17)$ than dexamethasone $(\log p \sim 1.74)$ and therefore should be preferentially incorporated/partitioned in the liposome membranes, leading to a decrease in dexamethasone encapsulation. The rank order of dexamethasone release from the nonextruded liposomes was similar to that from the extruded liposomes at both $37^{\circ} \mathrm{C}$ and $25^{\circ} \mathrm{C}$. Release profiles from DMPC and DPPC were similar at $37^{\circ} \mathrm{C}$. However, for nonextruded DPPC liposomes, heterogeneous distribution of dexamethasone reduced the main transition onset temperature even lower than that for the extruded liposomes. Nevertheless, dexamethasone release from nonextruded liposomes was slower than that from extruded liposomes at both 37 and $25^{\circ} \mathrm{C}$. Therefore, it would appear that the rate-controlling step for release of dexamethasone (to bulk) is diffusion across the multilamellar structure of the nonextruded liposomes [21].

Conventional chronic and acute treatments for osteoarthritis (OA) are by oral NSAIDs (such as diclofenac) and intra-articular injected glucocorticosteroids (such as dexamethasone) [55]. In free form, diclofenac and dexamethasone generate severe adverse effects with risks of toxicity; local injections of liposomal formulations for diclofenac and dexamethasone (each alone and their combination) were investigated to reduce these drawbacks. Employing RIA and immunoblot assay techniques, it was verified that the encapsulated drugs retained their biological activities: inhibitions of cyclooxygenases enzyme activity (diclofenac) and of cyclooxygenases protein expression (dexamethasone). A single intra-articular injection of each liposome-drug(s) formulation sufficed to reduce knee joint inflammation in OA rats over a timespan of 17 days using live-animal MRI.

\section{Hyaluronic Acid Delivery Systems}

Hyaluronic acid (HA), a polysaccharide consisting of a long chain of disaccharide, is a natural component of cartilage and plays a fundamental role in the maintenance of the tropic status of the cartilage. The various preparations of HA from different sources and molecular weights are widely used for more than 30 years in the treatment of knee OA to provide a further decrease in pain and stiffness and increase physical function [56]. The Western Ontario and McMaster Universities Osteoarthritis Index (WOMAC) has been used as a validated tool to assess the improvements in knee OA. The formulations include high molecular weight HA (HMW-HA) and low molecular weight HA (LMW-HA), but most studies demonstrated an improvement in all three variables from baseline, independent of the molecular weight HA used [57].

Intra-articular hyaluronic acid (HA) has been proposed as an alternative to steroids and nonsteroidal antiinflammatory drugs (NSAIDs) for the intra-articular treatment of OA. In conclusion, this controlled comparative study suggests that intra-articular hyaluronic acid of MW $500-730 \mathrm{kDa}$ (Hyalgan) exerts beneficial effects. As leading to a reduction of synovial inflammation and a slowing cartilage damage progression, comparative study confirms its validity as an alternative (not only symptomatological but also structural) to intra-articular steroids and NSAIDs in the treatment of OA of the knee [58].

Bannuru et al. compared the efficacy of intra-articular hyaluronic acid with corticosteroids for knee osteoarthritis (OA) and found that intra-articular corticosteroids appear to be relatively more effective for pain than intra-articular hyaluronic acid from baseline to week 4 . By week 4 , the 2 approaches have equal efficacy. However, hyaluronic acid has greater efficacy beyond week 8 . Understanding this trend is useful to clinicians when treating knee OA [59]. 
As Hyal clearance is extremely rapid and degradation quick in vivo by enzymatic or hydrolytic reactions, localisation of response is likely to be a problem, which takes place in biological environments [60].

5.1. Polymer Particles. Hyaluronic acid (HA) is a physiologic component of the synovial fluid and is reduced in OA joints. Therefore, due to its viscoelastic properties and protective effect on articular cartilage and soft tissue surfaces of joints, intra-articular injection of HA can restore the normal articular homoeostasis. These effects are evident when HA is properly administered into the articular space; therefore, the use of "image-guided" infiltration techniques is mandatory [61].

In the preliminary study, the $\mathrm{HA} / \mathrm{Col}$ II microspheres have shown to provide favorable ECM characteristics, with appropriate mechanical strength, and exhibited a 3D inclination. By injecting an HA/Col II mixture solution through a pair of HCPDEs under the influence of a high electrostatic field, microspheres were readily prepared with good sphericity and narrow size distribution. The watery microspheres were about $500-600 \mu \mathrm{m}$ in size and able to retain their nontoxicity upon treatments, such as gelation, crosslinking, and thermal treatment [62].

5.2. Hydrogels. Hydrogels are three-dimensional, hydrophilic, polymeric networks which are composed of homopolymers or copolymers and capable of imbibing large amounts of water or biological fluids. Due to the presence of chemical crosslinks (tie points, junctions) or physical crosslinks, such as entanglements or crystallites, they are insoluble but can swell in water [63].

A 50\% crosslinked Hyal hydrogel (Hyal 50\%) was synthesized in order to overcome the problem of rapid clearance of the polysaccharide hyaluronic acid (Hyal) in the treatment of osteoarthritis (OA). The $50 \%$ refers to the amount of $\mathrm{COOH}$ groups of the polysaccharide involved in the crosslinking reaction, that is, $50 \%$ of the total amount. We decided to make the polysaccharide water insoluble by crosslinking it in order to obtain a longer effect of Hyal in the action site (joint) and consequently to reduce the risk of infection due to numerous injections. The result of this reaction was the formation of a hydrogel.

Moreover, the local pharmacological treatment with hyaluronic acid has also been claimed to be a useful tool in OA treatment. Now, the Hyal 50\% hydrogel treatment seems to improve chondrocytes density and matrix appearance. Without evidence of tissue reaction or inflammation in the rabbit OA model, hydrogel tends to produce a smoother and more regular articular surface for a long period of time (50 days).

\section{Chondroitin Sulfate Delivery Systems}

Chondroitin sulfate (CS) is a safe and tolerable therapeutic agent for the management of OA whose effects include benefits that are not achieved by current medicines and include chondroprotection and the prevention of joint space narrowing [64-66]. CS is effective for the treatment of $\mathrm{OA}$, at least in part, and its therapeutic benefits occur through three main mechanisms [67]: (1) stimulation of extracellular matrix production by chondrocytes; (2) suppression of inflammatory mediators; (3) inhibition of cartilage degeneration. These in vitro findings motivate the consideration of CS for intra-articular therapeutic injections for the treatment of painful joint degenerative diseases and as a potential prophylactic against the progression of cartilage degeneration.

There has been growing interests in potential chondroprotective agent chondroitin sulfate (CS), a kind of natural element that forms the cartilage extracellular matrix [68]. From the analysis, chondroitin is found to be effective in improving the visual analog scale pain, mobility, and responding status of OA patients [69]. The safety of CS is excellent. However, bioavailability of CS is only in the region of $10-15 \%$. CS was used widely to treat OA via oral administration with mixed results $[70,71]$. The biological effects of sulfated glycosaminoglycans have been widely studied for their potential therapeutic benefits.

The anti-inflammatory and antiapoptotic effects of chondroitin sulfate (CS) are increasingly used to treat osteoarthritis [72]. Furthermore, several studies have described the benefits of chondroitin sulfate in applications for cartilage tissue engineering. The therapeutic effects of glucosamine and chondroitin sulfate in OA have been studied for 20 years, and their symptom relieving efficacies were recently analyzed by high-quality meta-analysis [73] .

6.1. Liposomes. Chondroitin sulphate is a sulphated GAG composed of a long unbranched polysaccharide chain with a repeating disaccharide structure of $\mathrm{N}$-acetylgalactosamine and glucuronic acid. The in vitro studies demonstrated that CS has both anabolic effects on cartilage metabolism and anticatabolic properties [74]. Several studies have shown clinical benefits of CS administration in patients with OA [75-77]. Moreover, in the case of arthritic diseases, it has been reported that the intra-articular administration of antiinflammatory drugs encapsulated in liposomes shows prolonged residence in the joint and reduction of inflammation.

Liposomes have been proposed as a means to target intraarticularly injected anti-inflammatory agents to phagocytic cells in inflamed synovial joints [78]. Liposomes prepared from naturally occurring biodegradable and nontoxic lipids are good candidates for local delivery of therapeutic agents. Treatment of arthritis by intra-articular administration of anti-inflammatory drugs encapsulated in liposomes prolongs the residence time of the drug in the joint [79]. In order to use liposome-entrapped chondroitin sulphate in the local treatment of inflammatory disorders, Trif et al. [43] studied ultrastructural characterization of liposomesentrapped chondroitin sulphate and proved their in vitro biocompatibility in a human dermal fibroblast culture system. Results indicated that appreciable cytotoxic effects were not induced by chondroitin sulphate, empty liposomes, and liposome-chondroitin sulphate systems, and cells maintain normal morphology when compared to control fibroblasts. 
6.2. Hydrogels. Hydrogels have been used extensively in the development of the "SMART" drug delivery systems ("SMART" drug delivery system (SDDS) is the multitargeted, $\mathrm{pH}$ responsive, stimuli-sensitive delivery systems, which are capable of self-regulation, integrated sensing, monitoring, and remote activation). A hydrogel is a network of hydrophilic polymers that can swell in water and hold a large amount of water while maintaining the structure. A three-dimensional network is formed by crosslinking polymer chains which can be provided by covalent bonds, hydrogen bonding, van der Waals interactions, or physical entanglements [80].

Specifically, neutral poly(ethylene glycol) (PEG) hydrogels were employed where negatively charged chondroitin sulfate $(\mathrm{ChS})$, one of the main extracellular matrix components of cartilage, was systematically incorporated into the PEG network at $0 \%, 20 \%$, or $40 \%$ to control the fixed charge density [81]. J. H. Hui [68] selected five types of hydrogel carriers (a-CD-EG 4400, a-CD-EG 8400, a-CD-EG 13300, a-CD-PEG 20000, and a-CD-PEG 35000). According to the biocompatibility studies, they found that no sign of redness or swelling around the knee was observed on days 3,7 , and 21 after injection before the rabbits were killed with $0.5 \mathrm{~mL}$ of the three kinds of hydrogel or normal saline in the knee joints. Combining the results from the in vitro and in vivo studies, a-CD-EG4400 was chosen as the carrier of CS in the treatment of joint defect in rabbits due to its ability to remain viable for the longest period of time while slowly retaining and releasing CS. Intra-articular injection of CS $(100 \mathrm{mg} / \mathrm{mL})$ carried by a-CD-EG 4400 hydrogel was effective in postponing osteoarthritis in rabbits by improving both the biomechanical and histological properties of the knee joints.

\section{Conclusions}

The ability to deliver highly therapeutic agents to diseased sites specifically is crucial for effectively treating all human illnesses. Intra-articular drug delivery can be a good means of targeting drug to the large joints (knees and hips) and has been expected to gain prominence for several reasons, such as only a minimum amount of drug is required to exert the desired pharmacological activity. A key requirement for drug delivery is demonstration of an ability to provide for increased intra-articular drug concentrations and residence time following administration to the joint space, as well as decreased systemic exposure to the drug. Many factors that influence the efficacy of a drug delivery vehicle for promoting sustained intra-articular residence time have been presented by several reviews, including particle size, vehicle safety, hydrophobicity, and charge.

However, successful commercialisation of any drug delivery technique requires careful consideration of a number of parameters particularly in elderly patients, including efficiency, cost, potential for toxicity, retention inside the body, sustainability of drug concentrations, and drug elimination kinetics [82]. The delivery techniques being investigated currently are paid little attention to many of these criteria, and any future endeavours will need to address these deficiencies before these techniques can become clinically exploitable.

\section{References}

[1] E. C. Sayre, L. C. Li, J. A. Kopec et al., "The effect of disease site (knee, hip, hand, foot, lower back or neck) on employment reduction due to osteoarthritis," Plos One, vol. 5, no. 5, pp. 17,2010.

[2] O. Sangha, "Epidemiology of rheumatic diseases," Rheumatology, vol. 39, no. 2, pp. 3-12, 2000.

[3] D. J. Hunter, J. J. McDougall, and F. J. Keefe, "The symptoms of osteoarthritis and the genesis of pain," Medical Clinics of North America, vol. 93, no. 1, pp. 83-100, 2009.

[4] M. Fransen, S. McConnell, G. Hernandez-Molina, and S. Reichenbach, "Does land-based exercise reduce pain and disability associated with hip osteoarthritis? A meta-analysis of randomized controlled trials," Osteoarthritis and Cartilage, vol. 18, no. 5, pp. 613-620, 2010.

[5] S. S. Bansal, A. Joshi, and A. K. Bansal, "New dosage formulations for targeted delivery of cyclo-oxygenase-2 inhibitorsfocus on use in the elderly," Drugs and Aging, vol. 24, no. 6, pp. 441-451, 2007.

[6] R. C. Lawrence, C. G. Helmick, F. C. Arnett et al., "Estimates of the prevalence of arthritis and selected musculoskeletal disorders in the United States," Arthritis and Rheumatism, vol. 41, no. 5, pp. 778-799, 1998.

[7] I. Elron-Gross, Y. Glucksam, D. Melikhov, and R. Margalit, "Cyclooxygenase inhibition by diclofenac formulated in bioadhesive carriers," Biochimica et Biophysica ActaBiomembranes, vol. 1778, no. 4, pp. 931-936, 2008.

[8] K. D. Allen, S. B. Adams, and L. A. Setton, "Evaluating intraarticular drug delivery for the treatment of osteoarthritis in a rat model," Tissue Engineering Part B, vol. 16, no. 1, pp. 81-92, 2010.

[9] G. Kogan, L. Šoltés, R. Stern, and P. Gemeiner, "Hyaluronic acid: a natural biopolymer with a broad range of biomedical and industrial applications," Biotechnology Letters, vol. 29, no. 1, pp. 17-25, 2007.

[10] S. H. R. Edwards, M. A. Cake, G. Spoelstra, and R. A. Read, "Biodistribution and clearance of intra-articular liposomes in a large animal model using a radiographic marker," Journal of Liposome Research, vol. 17, no. 3-4, pp. 249-261, 2007.

[11] P. M. Mountziaris, P. R. Kramer, and A. G. Mikos, "Emerging intra-articular drug delivery systems for the temporomandibular joint," Methods, vol. 47, no. 2, pp. 134-140, 2009.

[12] G. Leone, M. Fini, P. Torricelli, R. Giardino, and R. Barbucci, "An amidated carboxymethylcellulose hydrogel for cartilage regeneration," Journal of Materials Science: Materials in Medicine, vol. 19, no. 8, pp. 2873-2880, 2008.

[13] X. Chevalier, "Intraarticular treatments for osteoarthritis: new perspectives," Current Drug Targets, vol. 11, no. 5, pp. 546-560, 2010.

[14] M. Tunçay, S. Çaliş, H. S. Kaş, M. T. Ercan, I. Peksoy, and A. A. Hincal, "In vitro and in vivo evaluation of diclofenac sodium loaded album in microspheres," Journal of Microencapsulation, vol. 17, no. 2, pp. 145-155, 2000.

[15] M. Tunçay, S. Çaliş, H. S. Kaş, M. T. Ercan, I. Peksoy, and A. A. Hincal, "Diclofenac sodium incorporated PLGA (50:50) microspheres: formulation considerations and in vitro/in vivo evaluation," International Journal of Pharmaceutics, vol. 195, no. 1-2, pp. 179-188, 2000. 
[16] Y. Shi and G. Huang, "Recent developments of biodegradable and biocompatible materials based micro/nanoparticles for delivering macromolecular therapeutics," Critical Reviews in Therapeutic Drug Carrier Systems, vol. 26, no. 1, pp. 29-84, 2009.

[17] N. K. Green, C. W. Herbert, S. J. Hale et al., "Extended plasma circulation time and decreased toxicity of polymer-coated adenovirus," Gene Therapy, vol. 11, no. 16, pp. 1256-1263, 2004.

[18] H. Tamber, P. Johansen, H. P. Merkle, and B. Gander, "Formulation aspects of biodegradable polymeric microspheres for antigen delivery," Advanced Drug Delivery Reviews, vol. 57, no. 3, pp. 357-376, 2005.

[19] J. W. McGinity and P. B. O’Donnell, "Preparation of microspheres by the solvent evaporation technique," Advanced Drug Delivery Reviews, vol. 28, no. 1, pp. 25-42, 1997.

[20] M. Trie, C. Guillen, D. M. Vaughan et al., "Liposomes as possible carriers for lactoferrin in the local treatment of inflammatory diseases," Experimental Biology and Medicine, vol. 226, no. 6, pp. 559-564, 2001.

[21] U. Bhardwaj and D. J. Burgess, "Physicochemical properties of extruded and non-extruded liposomes containing the hydrophobic drug dexamethasone," International Journal of Pharmaceutics, vol. 388, no. 1-2, pp. 181-189, 2010.

[22] H. M. Burt, A. Tsallas, S. Gilchrist, and L. S. Liang, "Intraarticular drug delivery systems: overcoming the shortcomings of joint disease therapy," Expert Opinion on Drug Delivery, vol. 6, no. 1, pp. 17-26, 2009.

[23] M. R. Mozafari, "Liposomes: an overview of manufacturing techniques," Cellular and Molecular Biology Letters, vol. 10, no. 4, pp. 711-719, 2005.

[24] M. R. Mozafari, "Liposomes: an overview of manufacturing techniques," Cellular and Molecular Biology Letters, vol. 10, no. 4, pp. 711-719, 2005.

[25] O. Wichterle and D. Lím, "Hydrophilic gels in biologic use," Nature, vol. 185, no. 4706, pp. 117-118, 1960.

[26] A. S. Hoffman, "Hydrogels for biomedical applications," Advanced Drug Delivery Reviews, vol. 54, no. 1, pp. 3-12, 2002.

[27] F. Li, G. Wu, J. Wang, and C. Wang, "Tribological properties of poly(vinyl alcohol) hydrogel in response to ceramic femoral component," Iranian Polymer Journal, vol. 18, no. 11, pp. 881890, 2009.

[28] L. Yu and J. Ding, "Injectable hydrogels as unique biomedical materials," Chemical Society Reviews, vol. 37, no. 8, pp. 14731481, 2008.

[29] N. A. Peppas and A. R. Khare, "Preparation, structure and diffusional behavior of hydrogels in controlled release," Advanced Drug Delivery Reviews, vol. 11, no. 1-2, pp. 1-35, 1993.

[30] M. Hamidi, A. Azadi, and P. Rafiei, "Hydrogel nanoparticles in drug delivery," Advanced Drug Delivery Reviews, vol. 60, no. 15, pp. 1638-1649, 2008.

[31] C. P. Duffy, C. J. Elliott, R. A. O’Connor et al., "Enhancement of chemotherapeutic drug toxicity to human tumour cells in vitro by a subset of non-steroidal anti-inflammatory drugs (NSAIDS)," European Journal of Cancer, vol. 34, no. 8, pp. 1250-1259, 1998.

[32] H. Heli, A. Jabbari, S. Majdi, M. Mahjoub, A. A. MoosaviMovahedi, and S. Sheibani, "Electrooxidation and determination of some non-steroidal anti-inflammatory drugs on nanoparticles of Ni-curcumin-complex-modified electrode," Journal of Solid State Electrochemistry, vol. 13, no. 12, pp. 1951-1958, 2009.
[33] J. M. Bjordal, A. E. Ljunggren, A. Klovning, and L. Slørdal, "Non-steroidal anti-inflammatory drugs, including cyclooxygenase-2 inhibitors, in osteoarthritic knee pain: metaanalysis of randomised placebo controlled trials," British Medical Journal, vol. 329, no. 7478, pp. 1317-1320, 2004.

[34] J. Hippisley-Cox and C. Coupland, "Risk of myocardial infarction in patients taking cyclo-oxygenase-2 inhibitors or conventional non-steroidal anti-inflammatory drugs: population based nested case-control analysis," British Medical Journal, vol. 330, no. 7504, pp. 1366-1369, 2005.

[35] A. Fernández-Carballido, R. Herrero-Vanrell, I. T. MolinaMartínez, and P. Pastoriza, "Biodegradable ibuprofen-loaded PLGA microspheres for intraarticular administration: effect of Labrafil addition on release in vitro," International Journal of Pharmaceutics, vol. 279, no. 1-2, pp. 33-41, 2004.

[36] S. Y. Lin, K. S. Chen, H. H. Teng, and M. J. Li, "In vitro degradation and dissolution behaviours of microspheres prepared by three low molecular weight polyesters," Journal of Microencapsulation, vol. 17, no. 5, pp. 577-586, 2000.

[37] P. A. Todd and E. M. Sorkin, "Diclofenac sodium. A reappraisal of its pharmacodynamic and pharmacokinetic properties, and therapeutic efficacy," Drugs, vol. 35, no. 3, pp. 244285, 1988.

[38] R. N. Brogden, R. C. Heel, G. E. Pakes, and G. S. Avery, "Diclofenac sodium: a review of its pharmacological properties and therapeutic use in rheumatic diseases and pain of varying origin,” Drugs, vol. 20, no. 1, pp. 24-48, 1980.

[39] M. Saravanan, K. Bhaskar, G. Maharajan, and K. S. Pillai, "Development of gelatin microspheres loaded with diclofenac sodium for intra-articular administration," Journal of Drug Targeting, vol. 19, no. 2, pp. 96-108, 2010.

[40] M. Saravanan, J. Anbu, G. Maharajan, and K. S. Pillai, "Targeted delivery of diclofenac sodium via gelatin magnetic microspheres formulated for intra-arterial administration," Journal of Drug Targeting, vol. 16, no. 5, pp. 366-378, 2008.

[41] H. P. Thakkar and R. R. Murthy, "Effect of cross-linking agent on the characteristics of celecoxib loaded chitosan microspheres," Asian Journal of Pharmaceutics, vol. 2, no. 4, pp. 246-251, 2008.

[42] S. Türker, S. Erdoğan, A. Y. Özer et al., "Scintigraphic imaging of radiolabelled drug delivery systems in rabbits with arthritis," International Journal of Pharmaceutics, vol. 296, no. 1-2, pp. 34-43, 2005.

[43] M. Trif, L. Moldovan, M. Moisei, O. Craciunescu, and O. Zarnescu, "Liposomes-entrapped chondroitin sulphate: ultrastructural characterization and in vitro biocompatibility," Micron, vol. 39, no. 7, pp. 1042-1045, 2008.

[44] G. S. Canto, S. L. Dalmora, and A. G. Oliveira, "Piroxicam encapsulated in liposomes: characterization and in vivo evaluation of topical anti-inflammatory effect," Drug Development and Industrial Pharmacy, vol. 25, no. 12, pp. 1235-1239, 1999.

[45] I. Elron-Gross, Y. Glucksam, I. E. Biton, and R. Margalit, "A novel Diclofenac-carrier for local treatment of osteoarthritis applying live-animal MRI," Journal of Controlled Release, vol. 135, no. 1, pp. 65-70, 2009.

[46] R. G. Gray and N. L. Gottlieb, "Intra-articular corticosteroids. An updated assessment," Clinical Orthopaedics and Related Research, vol. 177, pp. 235-263, 1983.

[47] R. M. Schiffelers, M. Banciu, J. M. Metselaar, and G. Storm, "Therapeutic application of long-circulating liposomal glucocorticoids in auto-immune diseases and cancer," Journal of Liposome Research, vol. 16, no. 3, pp. 185-194, 2006. 
[48] C. M. Spies, J. W. Bijlsma, G. R. Burmester, and F. Buttgereit, "Pharmacology of glucocorticoids in rheumatoid arthritis," Current Opinion in Pharmacology, vol. 10, no. 3, pp. 302-307, 2010.

[49] W. Jubiz and A. W. Meikle, "Alterations of glucocorticoid actions by other drugs and disease states," Drugs, vol. 18, no. 2, pp. 113-121, 1979.

[50] A. Berthold, K. Cremer, and J. Kreuter, "Collagen microparticles: carriers for glucocorticosteroids," European Journal of Pharmaceutics and Biopharmaceutics, vol. 45, no. 1, pp. 23-29, 1998.

[51] E. Horisawa, T. Hirota, S. Kawazoe et al., "Prolonged antiinflammatory action of DL-lactide/glycolide copolymer nanospheres containing betamethasone sodium phosphate for an intra-articular delivery system in antigen-induced arthritic rabbit," Pharmaceutical Research, vol. 19, no. 4, pp. 403-410, 2002.

[52] N. Butoescu, C. A. Seemayer, M. Foti, O. Jordan, and E. Doelker, "Dexamethasone-containing PLGA superparamagnetic microparticles as carriers for the local treatment of arthritis," Biomaterials, vol. 30, no. 9, pp. 1772-1780, 2009.

[53] N. Butoescu, O. Jordan, A. Petri-Fink, H. Hofmann, and E. Doelker, "Co-encapsulation of dexamethasone 21-acetate and SPIONs into biodegradable polymeric microparticles designed for intra-articular delivery," Journal of Microencapsulation, vol. 25, no. 5, pp. 339-350, 2008.

[54] V. A. Tsotas, S. Mourtas, and S. G. Antimisiaris, "Dexamethasone incorporating liposomes: effect of lipid composition on drug trapping efficiency and vesicle stability," Drug Delivery, vol. 14, no. 7, pp. 441-445, 2007.

[55] I. Elron-Gross, Y. Glucksam, and R. Margalit, "Liposomal dexamethasone-diclofenac combinations for local osteoarthritis treatment," International Journal of Pharmaceutics, vol. 376, no. 1-2, pp. 84-91, 2009.

[56] F. Atamaz, Y. Kirazli, and Y. Akkoc, "A comparison of two different intra-articular hyaluronan drugs and physical therapy in the management of knee osteoarthritis," Rheumatology International, vol. 26, no. 10, pp. 873-878, 2006.

[57] B. Scott, The benefits of intra-articular high molecular weight hyaluronic acid injections compared to low molecular weight intra-articular hyaluronic acid in the reduction of pain, disability, and stiffness in adult patients with osteoarthritis of the knee, M.S. thesis, School of Physician Assistant Studies, 2009.

[58] L. Frizziero and I. Pasquali Ronchetti, "Intra-articular treatment of osteoarthritis of the knee: an arthroscopic and clinical comparison between sodium hyaluronate (500-730 $\mathrm{kDa}$ ) and methylprednisolone acetate," Journal of Orthopaedics and Traumatology, vol. 3, no. 2, pp. 89-96, 2002.

[59] R. R. Bannuru, N. S. Natov, I. E. Obadan, L. L. Price, C. H. Schmid, and T. E. McAlindon, "Therapeutic trajectory of hyaluronic acid versus corticosteroids in the treatment of knee osteoarthritis: a systematic review and meta-analysis," Arthritis Care and Research, vol. 61, no. 12, pp. 1704-1711, 2009.

[60] R. Barbucci, S. Lamponi, A. Borzacchiello et al., "Hyaluronic acid hydrogel in the treatment of osteoarthritis," Biomaterials, vol. 23, no. 23, pp. 4503-4513, 2002.

[61] M. Abate, D. Pulcini, A. Di Iorio, and C. Schiavone, "Viscosupplementation with intra-articular hyaluronic acid for treatment of osteoarthritis in the elderly," Current Pharmaceutical Design, vol. 16, no. 6, pp. 631-640, 2010.
[62] S. J. Chang, S. M. Kuo, I. Manousakas, G. C. Niu, and J. P. Chen, "Preparation and characterization of hyaluronan/collagen II microspheres under an electrostatic field system with disc electrodes," Acta Biomaterialia, vol. 5, no. 1, pp. 101-114, 2009.

[63] N. A. Peppas, P. Bures, W. Leobandung, and H. Ichikawa, "Hydrogels in pharmaceutical formulations," European Journal of Pharmaceutics and Biopharmaceutics, vol. 50, no. 1, pp. 27-46, 2000.

[64] G. Verbruggen, S. Goemaere, and E. M. Veys, "Chondroitin sulfate: S/DMOAD (structure/disease modifying antiosteoarthritis drug) in the treatment of finger joint OA," Osteoarthritis and Cartilage, vol. 6, supplement A, pp. 37-38, 1998.

[65] M. Kobo, K. Ando, T. Mimura, Y. Matsusue, and K. Mori, "Chondroitin sulfate for the treatment of hip and knee osteoarthritis: current status and future trends," Life Sciences, vol. 85, no. 13-14, pp. 477-483, 2009.

[66] M. Cohen, R. Wolfe, T. Mai, and D. Lewis, "A randomized, double blind, placebo controlled trial of a topical cream containing glucosamine sulfate, chondroitin sulfate, and camphor for osteoarthritis of the knee," Journal of Rheumatology, vol. 30, no. 3, pp. 523-528, 2003.

[67] P. S. Chan, J. P. Caron, G. J. M. Rosa, and M. W. Orth, "Glucosamine and chondroitin sulfate regulate gene expression and synthesis of nitric oxide and prostaglandin $\mathrm{E}_{2}$ in articular cartilage explants," Osteoarthritis and Cartilage, vol. 13, no. 5, pp. 387-394, 2005.

[68] J. H. Hui, S. W. Chan, J. Li et al., "Intra-articular delivery of chondroitin sulfate for the treatment of joint defects in rabbit model," Journal of Molecular Histology, vol. 38, no. 5, pp. 483489, 2007.

[69] B. F. Leeb, H. Schweitzer, K. Montag, and J. S. Smolen, "A metaanalysis of chondroitin sulfate in the treatment of osteoarthritis," Journal of Rheumatology, vol. 27, no. 1, pp. 205-211, 2000.

[70] S. K. Tat, J. P. Pelletier, F. Mineau, N. Duval, and J. MartelPelletier, "Variable effects of 3 different chondroitin sulfate compounds on human osteoarthritic cartilage/chondrocytes: relevance of purity and production process," Journal of Rheumatology, vol. 37, no. 3, pp. 656-664, 2010.

[71] D. Uebelhart, E. J. M. Thonar, P. D. Delmas, A. Chantraine, and E. Vignon, "Effects of oral chondroitin sulfate on the progression of knee osteoarthritis: a pilot study," Osteoarthritis and Cartilage, vol. 6, supplement A, pp. 39-46, 1998.

[72] J. Egea, A. G. García, J. Verges, E. Montell, and M. G. López, "Antioxidant, antiinflammatory and neuroprotective actions of chondroitin sulfate and proteoglycans," Osteoarthritis and Cartilage, vol. 18, supplement 1, pp. S24-S27, 2010.

[73] S. C. Vlad, M. P. LaValley, T. E. McAlindon, and D. T. Felson, "Glucosamine for pain in osteoarthritis: why do trial results differ?" Arthritis and Rheumatism, vol. 56, no. 7, pp. 22672277, 2007.

[74] A. Fioravanti and G. Collodel, "In vitro effects of chondroitin sulfate," Advances in Pharmacology, vol. 53, pp. 449-465, 2006.

[75] V. Prabhakar and R. Sasisekharan, "The biosynthesis and catabolism of galactosaminoglycans," Advances in Pharmacology, vol. 53, pp. 69-115, 2006.

[76] D. O. Clegg, D. J. Reda, C. L. Harris et al., "Glucosamine, chondroitin sulfate, and the two in combination for painful 
knee osteoarthritis," The New England Journal of Medicine, vol. 354, no. 8, pp. 795-808, 2006.

[77] D. Uebelhart, M. Malaise, R. Marcolongo et al., "Intermittent treatment of knee osteoarthritis with oral chondroitin sulfate: a one-year, randomized, double-blind, multicenter study versus placebo," Osteoarthritis and Cartilage, vol. 12, no. 4, pp. 269-276, 2004.

[78] J. Mönkkönen, J. Liukkonen, M. Taskinen, T. D. Heath, and A. Urtti, "Studies on liposome formulationsfor intra-articular delivery of clodronate," Journal of Controlled Release, vol. 35, no. 2-3, pp. 145-154, 1995.

[79] A. S. Williams, J. P. Camilleri, R. M. Goodfellow, and B. D. Williams, "A single intra-articular injection of liposomally conjugated methotrexate suppresses joint inflammation in rat antigen-induced arthritis," British Journal of Rheumatology, vol. 35, no. 8, pp. 719-724, 1996.

[80] Y. Qiu and K. Park, "Environment-sensitive hydrogels for drug delivery," Advanced Drug Delivery Reviews, vol. 53, no. 3, pp. 321-339, 2001.

[81] I. Villanueva, S. K. Gladem, J. Kessler, and S. J. Bryant, “Dynamic loading stimulates chondrocyte biosynthesis when encapsulated in charged hydrogels prepared from poly(ethylene glycol) and chondroitin sulfate," Matrix Biology, vol. 29, no. 1, pp. 51-62, 2010.

[82] S. S. Bansal, A. Joshi, and A. K. Bansal, "New dosage formulations for targeted delivery of cyclo-oxygenase-2 inhibitors: focus on use in the elderly," Drugs and Aging, vol. 24, no. 6, pp. 441-451, 2007. 

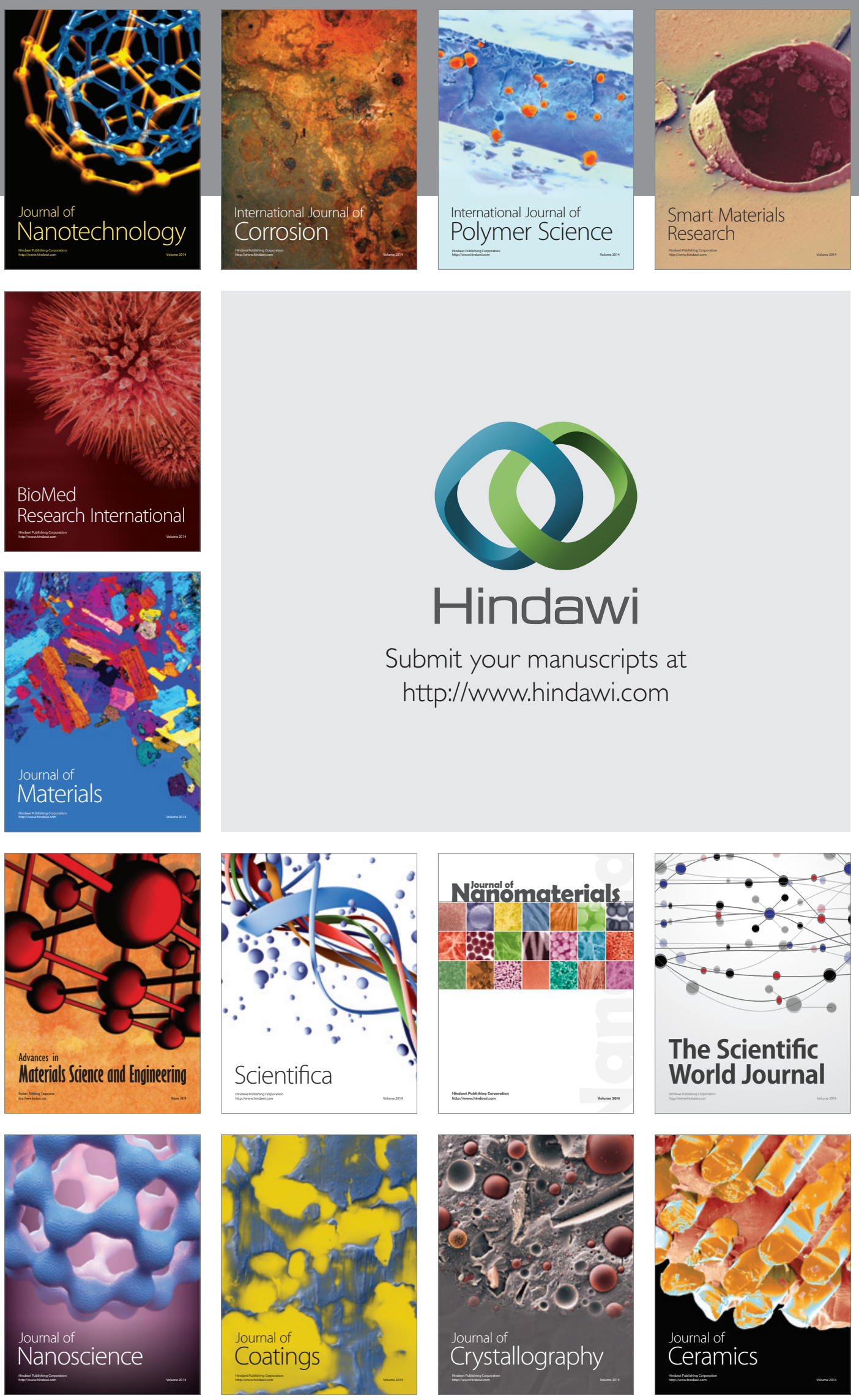

The Scientific World Journal

Submit your manuscripts at

http://www.hindawi.com

\section{World Journal}

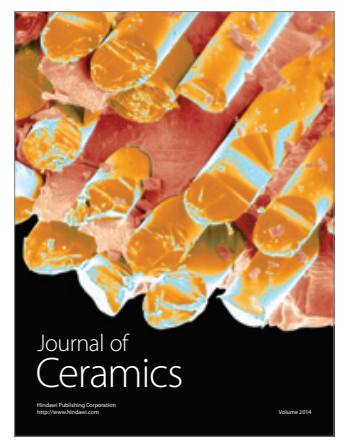

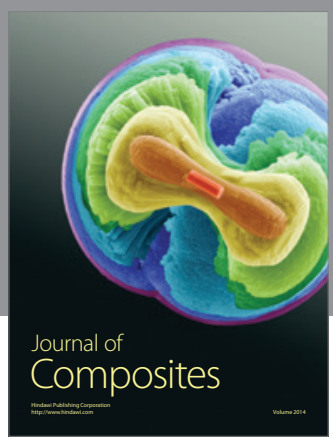
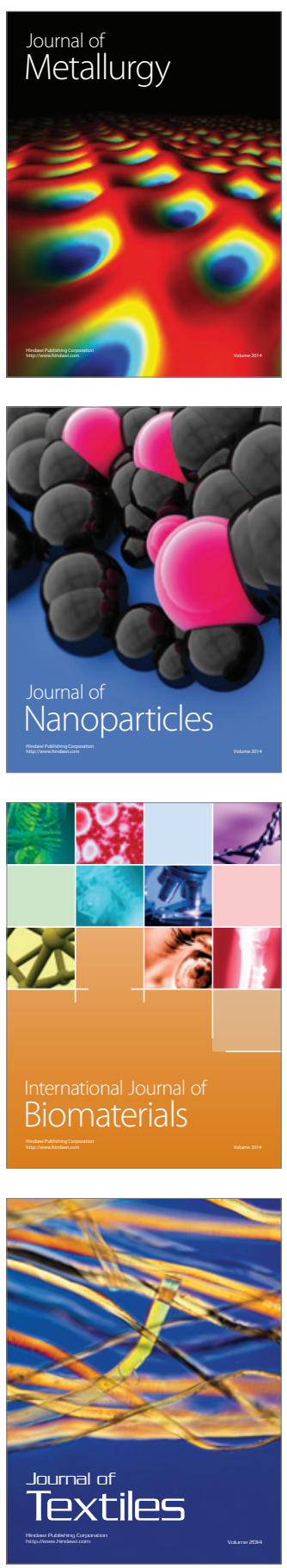